\title{
Evidence for an Innate Immune Response in the Immature Human Intestine: Toll-Like Receptors on Fetal Enterocytes
}

\author{
ROBERT D. FUSUNYAN, NANDA N. NANTHAKUMAR, MANUEL E. BALDEON, AND \\ W. ALLAN WALKER \\ Developmental Gastroenterology Laboratory, Combined Program in Pediatric Gastroenterology and \\ Nutrition, Massachusetts General Hospital, Harvard Medical School, Charlestown, Massachusetts \\ 02129-2060, U.S.A.
}

\begin{abstract}
ABST
The intestinal epithelium is an active participant in the mu-
cosal immune response against luminal pathogens. Microorgan-
isms and their cell wall products, i.e. lipopolysaccharide (LPS),
can stimulate the enterocyte to produce an innate immune re-
sponse with the increased production of IL- 8 via an activation of
the transcription factor NF $\kappa$ B. The innate response mechanism,
however, has not been understood until the recent description of
a family of human toll-like receptors (hTLR) on immune cells
that interact with LPS and modulate the IL-8 response via an
intracellular signal transduction pathway similar to that of the
IL-1 receptor family. Accordingly, in this study we have sought
to determine the constitutive and regulated expression of hTLR
on a nonmalignant human fetal primary small intestinal cell line
(H4 cells) and on small intestinal samples of ileum from human
fetuses (age $18-21$ wk). Specimens were examined by reverse-
transcription PCR, Western blot analysis, and immunofluores-
cence for hTLR2 and hTLR4 mRNA and protein and to deter-
mine whether their expression was regulated by LPS or by an
endogenous inflammatory stimulus, IL-1 $\beta$. hTLR2 and hTLR4
\end{abstract}
The mucosal surface of the gastrointestinal tract interfaces with the complex environment of the gut lumen, which contain an extensive variety of both commensal and pathogenic microbes. To contain this bacterial antigenic load, the mucosal epithelium has evolved features allowing it to function as an active participant in mucosal immune responses as well as a physical barrier to the uptake of noxious substances (1). When

Received October 27, 2000; accepted December 14, 2000.

Correspondence: W. Allan Walker, M.D., Developmental Gastroenterology Laboratory, Combined Program in Pediatric Gastroenterology and Nutrition, Massachusetts General Hospital-East, 149 13th St. (149-3404), Charlestown, MA 02129-2060, U.S.A.: e-mail: walker@helix.mgh.harvard.edu

Supported by National Institute of Health grants K08 DK02481, P01 DK33506, R37 HD12437, and R01 HD31852, and a grant from Wyeth Nutritionals, Inc. were expressed constitutively on $\mathrm{H} 4$ cells and on human fetal small intestinal enterocytes, predominantly on the basolateral surface of crypt enterocytes. Inflammatory stimuli appeared to regulate hTLR transcription (IL-1 $\beta$ increased both hTLR2 and hTLR4 whereas LPS decreased hTLR4) and possibly translation (qualitative observations). The presence of hTLR on human fetal enterocyte suggests a mechanism for the innate immune response to pathogens and could provide the basis for further study of the accentuated inflammatory response in age-dependent gastrointestinal diseases such as necrotizing enterocolitis. (Pediatr Res 49: 589-593, 2001)

Abbreviations
hTLR, human toll-like receptors
H4 cells, human nonmalignant fetal enterocyte cell line
THP-1, human monocyte cell line
PAMP, pathogen-associated molecular pattern
PRR, pattern recognition receptors

microbes interact with the gut epithelium, the gut can react by evoking both an immediate and innate as well as a delayed, adaptive immune response to contain their penetration. This interaction is termed "microbial-epithelial crosstalk" $(2,3)$. The innate immune response represents a genetically conserved immune response across species against pathogens and their cellular products, termed PAMP, which are recognized by PRR on immune cells (macrophages, monocytes, etc.) (4). This innate response can evoke either phagocytosis, a release of antibacterial peptides (defensins), or can stimulate secretion of inflammatory cytokines [IL-8, IL-6, tumor necrosis factoralpha (TNF- $\alpha)$, etc.] $(5,6)$. The cell wall component lipopolysaccharide (LPS) of Gram-negative organisms represents the prototypic PAMP and has been studied extensively because it 
accounts for the devastating fatal disease septic shock $(7,8)$. Previous studies have shown that LPS is opsonized by an LPS binding protein (LBP) found in serum and luminal secretions and this LPS-LBP complex facilitates its binding to a glycosylphosphatidylinositol (GPI)-anchored receptor, CD14, on the surface of immune cells (9). Because CD14 is a surface protein without a cytoplasmic domain and therefore unable to transmit signals to activate $\mathrm{NF} \kappa \mathrm{B}$ after interaction with the LBP-LPS complex, the cellular mechanism of the innate immune response to LPS has heretofore been unknown.

Recently, a family of PRR that recognized LPS and other bacterial products (PAMP) have been identified on rodent and human monocytes (10) that have a signal transduction pathway for the stimulation of proinflammatory cytokine genes (4, 10-12). These PRR possess type 1 transmembrane proteins with a leucine-rich repeat extracellular domain and a cytoplasmic domain with homology to the mammalian IL-1 receptor (IL-1R) family. These receptors, termed the hTLR family, are the mammalian homologue to the Drosophila receptor protein called toll, which is involved in developmental regulation of dorsoventral polarity in fly embryos and innate immunity in adult flies (11). To date, 10 hTLR have been identified $(13,14)$ but only two have known ligands. hTLR2 and hTLR4 have been shown to respond to LPS and other PAMPs, e.g. peptidoglycans of most bacterial organisms $(15,16)$, and to activate $\mathrm{NF} \kappa \mathrm{B}$ resulting in increased production of IL-8 and other inflammatory cytokines (17).

Furthermore, Cario et al. (18) have shown that mRNA and proteins for hTLR 2 and hTLR4 are constitutively expressed on human intestinal cancer cell lines (Caco-2, HT-29, and T-84). This observation has helped to explain the mechanism of the innate immune response to LPS by a nonclassical immune cell, i.e. the enterocyte. We have recently reported that LPS and IL- $1 \beta$, an endogenous inflammatory stimulant, can cause an accentuated innate response (IL-8 secretion) by enterocytes from human fetuses compared with older children (19). Accordingly, in this study we have extended this observation to determine whether the immature human small intestine expressed hTLR on their surface using a nonmalignant human fetal primary small intestinal cell line ( $\mathrm{H} 4$ cells) and human fetal ileal intestinal samples. If present, their expression may help explain the mechanism of innate immunity to PAMP, such as LPS, after interaction with the immature enterocyte. Furthermore, the presence of these receptors on immature enterocytes may provide insight into the mechanism of the accentuated inflammatory response noted in age-dependent gastrointestinal disease states such as necrotizing enterocolitis (19).

\section{METHODS}

Cell lines. A human fetal nonmalignant primary small intestinal cell line, designated $\mathrm{H} 4$ cells (20-34 passages), has been characterized by our laboratory (20) and was used to represent immature fetal enterocytes. A human monocyte cell line, THP-1 cells (TIB 202, ATCC, Manassas, VA, U.S.A.), was used as positive controls. H4 and THP-1 cells were plated into T-75 flasks (Falcon, Franklin Lakes, NJ, U.S.A.) in an initial cell density of $5 \times 10^{4}$ cells $/ \mathrm{cm}^{2}$. H4 cells were grown in Dulbecco's modified Eagle's medium (GIBCO, Grand Island, NY, U.S.A.) supplemented with $5 \%$ fetal bovine serum (FBS) (Biowhittaker, Walkersville, MD, U.S.A.), $2 \mathrm{mM}$ glutamine (GIBCO), 100,000 U/L penicillin, and $100 \mathrm{mg} / \mathrm{L}$ streptomycin (GIBCO). THP-1 cells were grown in RPMI 1640 media (ATCC), containing 10\% FBS (Biowhittaker), $1 \mathrm{mM}$ sodium pyruvate (GIBCO), $2 \mathrm{mM}$ L-glutamine (GIBCO), 4.5 $\mathrm{g} / \mathrm{L}$ glucose, $1.5 \mathrm{~g} / \mathrm{L}$ sodium bicarbonate, $10 \mathrm{mM}$ HEPES (GIBCO), and $0.05 \mathrm{mM}$ 2-mecaptoethanol (Sigma Chemical Co., St. Louis, MO, U.S.A.). The cells were cultured in a humid atmosphere of $95 \% \mathrm{O}_{2} / 5 \% \mathrm{CO}_{2}$ at $37^{\circ} \mathrm{C}$ as described (19). H4 cells, upon reaching confluence, were incubated in control media or in media containing LPS $(100 \mu \mathrm{g} / \mathrm{mL})$ or IL-1 $\beta(1 \mathrm{ng} / \mathrm{mL})$ for $24 \mathrm{~h}$. The expression of hTLR2 and hTLR4 was then determined by RT-PCR analysis, Western blot analysis, and immunofluorescence.

Fetal intestinal samples. Human small intestinal ileal samples were obtained from prostaglandin/saline-induced aborted fetuses at the age of 18-21 wk with informed consent from all concerned parties according to the regulations of the Human Studies Committees at the Brigham and Women's Hospital and Massachusetts General Hospital. To maintain sterility, tissues were collected only from fetuses in which the abdomen had not been previously opened. Fetal ileal small intestinal samples were fixed in $4 \%$ paraformaldehyde for $2 \mathrm{~h}$ at $4^{\circ} \mathrm{C}$ before being "snap" frozen in liquid nitrogen for cryosectioning.

RNA isolation and reverse-transcription PCR. Total RNA was extracted from $\mathrm{H} 4$ and THP-1 cells by a single-step method of RNA isolation using an acid guanidinium thiocyanate-phenol-chloroform extraction $(18,19)$. The reversetranscription (RT)-PCR analysis was carried out with 1-2 $\mu \mathrm{g}$ of total RNA using oligo-dT/random primers, and specific cDNA products were amplified for 28 cycles using specific pairs of primers and conditions for hTLR2, hTLR4, and $\beta$-actin as described previously (18). For TLR2, the PCR primers were 5'-GCCAAAGTCTTGATTGATTGG-3' and 5'-TTGAAGTTCTCCAGCTCCTG-3' (21) and for TLR4 the primers were 5'-TGGATACGTTTCCTTATAAG-3' and 5'-GAAATGGAGGCACCCCTTC-3' (21). $\beta$-actin primeus were obtained from PROMEGA.

After the RT-PCR reaction, the products were resolved by electrophoresis in $1.5-2 \%$ agarose gels and visualized by ethidium bromide staining. The relative amounts of the PCR products were measured by densitometry and the ratio of hTLR to $\beta$-actin calculated.

Western blot analysis. H4 and THP-1 cells were lysed with extraction buffer (10 mM Tris $\mathrm{HCl}, \mathrm{pH} 8.0,150 \mathrm{mM} \mathrm{NaCl}, 1 \%$ NP40) containing aprotinin, leupeptin, and phenylmethylsulfonyl fluoride (Sigma Chemical Co.). Lysates were clarified by centrifugation at $4^{\circ} \mathrm{C}$ for $15 \mathrm{~min}$. at $16,000 \times \mathrm{g}$. Fifty micrograms of $\mathrm{H} 4$ cell lysates was heated and loaded onto SDSPAGE and then transferred onto nitrocellulose membranes. These membranes were then blocked with $5 \%$ nonfat milk in Tris-buffered saline (TBS) plus $0.1 \%$ IGEPAL CA 630 (Sigma Chemical Co.). The blots were incubated with rabbit antihTLR2 and anti-hTLR4 polyclonal antibodies for $1 \mathrm{~h}$ followed by incubation with anti-rabbit IgG-horseradish peroxidase 
(HRP)-linked antibody (Amersham Biotech, Piscataway, NJ, U.S.A.). The membranes were developed with an enhanced chemiluminescence detection kit (Pierce, Rockford, IL, U.S.A.). (Anti-hTLR2 and anti-hTLR4 antisera (18) were kindly provided by Drs. Elke Cario and Daniel K. Podolsky from the Gastrointestinal Unit of the Medical Service at the Massachusetts General Hospital, Boston, MA, U.S.A.)

Immunofluorescence. Immunofluorescence analysis for hTLR2 and hTLR4 was performed on H4 cells mounted onto glass slides and on frozen sections of ileal mucosa obtained from fetal intestine. Cells and tissue sections were first fixed in $2 \%$ paraformaldehyde in PBS for $10 \mathrm{~min}$ at $4^{\circ} \mathrm{C}$ followed by washing in PBS (GIBCO). After washing in PBS, tissue and cells were incubated with rabbit anti-hTLR2 or anti-hTLR4 antibody for $1 \mathrm{~h}$ and then washed in cold PBS. This was followed by incubation for 45 min with an anti-rabbit $\operatorname{IgG}$ linked to FITC (Jackson Immunoresearch Laboratory, West Grove, PA, U.S.A.). The tissues were counterstained with anti-cytokeratin (CK5) mouse MAb (Sigma Chemical Co.) followed by horse anti-mouse IgG linked to Texas Red (Jackson Laboratory). The slides were washed, mounted with Vectashield $Æ$ (Vector Laboratory, Burlingame, CA, U.S.A.) and viewed for immunofluorescence using a confocal microscope with a TCVS 4D scanner (Leica, Iceburg, Switzerland) connected to an inverted Leitz DM IRB microscope (Oberkonen, Germany). Images were processed using a TCS-NT software package.

Statistical analysis. Results, shown in Figure $1 B$, are presented as mean \pm SEM. Effects of treatment with LPS or IL- $1 \beta$ on hTLR2 and hTLR4 expression were analyzed by two-way ANOVA, and $p$ values $<0.05$ were considered significant.

\section{RESULTS}

hTLR2 and hTLR4 mRNA expression on H4 cells. H4 cells were incubated in either control media or media containing LPS $(100 \mu \mathrm{g} / \mathrm{mL})$ or IL-1 $\beta(1 \mathrm{ng} / \mathrm{mL})$ for $24 \mathrm{~h}$. Total mRNA was isolated from these cells and the levels of hTLR2 and hTLR4 mRNA expression determined by RT-PCR (Fig. 1A). hTLR2 and hTLR4 mRNA were noted to be expressed constitutively in $\mathrm{H} 4$ cells. Using densitometry to quantitate expression (Fig. $1 B$ ), exposure to IL- $1 \beta$ seemed to increase the expression of hTLR 2 mRNA but was not statistically significant ( $p>0.1$, IL-1 $\beta$ stimulated versus unstimulated,). LPS exposure had no effect. In contrast, hTLR4 expression seemed to decrease after LPS exposure but was also not statistically significant ( $p>0.4$, LPS stimulated versus unstimulated). IL-1 $\beta$ stimulation on hTLR4 expression mirrors that of hTLR2 and was similarly statistically not significant $(p>0.1, \mathrm{IL}-1 \beta$ stimulated versus unstimulated) (Fig. $1 B$ ). These patterns of expression, albeit not statistically significant, suggest that the level of mRNA expression of hTLR may be regulated by inflammatory stimuli, e.g. LPS and IL- $1 \beta$, in $\mathrm{H} 4$ cells.

hTLR2 and hTLR4 protein expression in $\mathrm{H} 4$ cells. The expression of hTLR 2 and hTLR4 proteins was determined by Western blot analysis. Both hTLR2 and hTLR4 proteins were constitutively expressed in $\mathrm{H} 4$ cells (Fig. 2). However, the level of expression was significantly lower than in THP-1 cells.

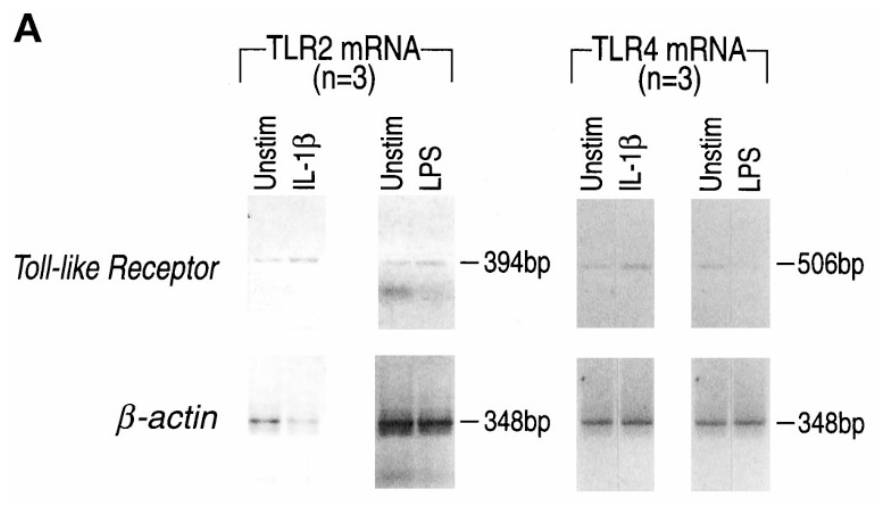

B

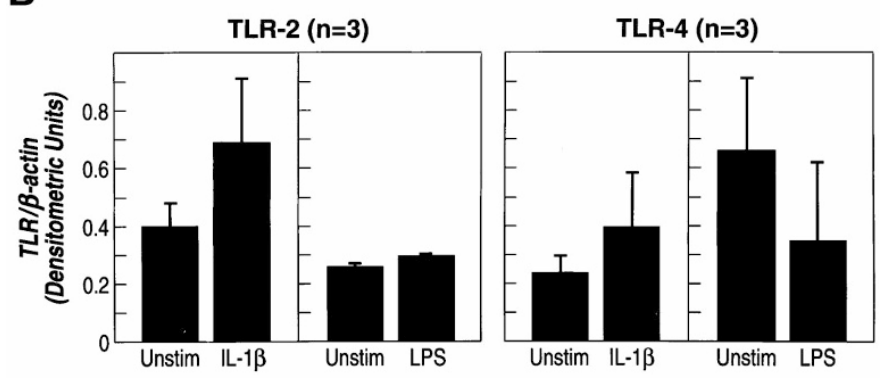

Figure 1. (A) hTLR2 mRNA and hTLR4 mRNA are expressed constitutively in $\mathrm{H} 4$ cells and seem to be modulated by exposure to LPS and IL-1 $\beta$. (B) After stimulation with IL-1 $\beta$, hTLR2 and hTLR4 mRNA accumulation in 44 cells is increased. After LPS stimulation, hTLR4 mRNA expression is decreased, whereas hTLR2 mRNA was unchanged. The RT-PCR results in $A$ are representative of three experiments and the graphic representation of the RT-PCR results in $B$ (expressed as a ratio of hTLR to $\beta$-actin) are the average means and SEM of three experiments.

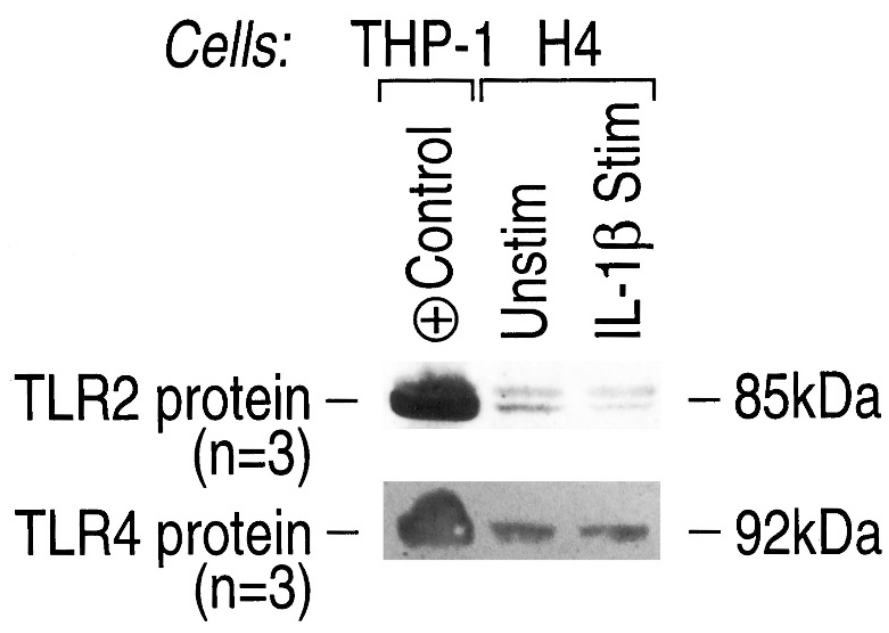

Figure 2. Both hTLR 2 and hTLR 4 proteins are constitutively expressed in $\mathrm{H} 4$ cells but their levels are much less than in THP-1 (positive control) cells. Upon stimulation by IL- $1 \beta$, hTLR2 protein seemed to be down-regulated and hTLR4 unchanged. Results were representative of three Western blot analyses

Upon stimulation with IL-1 $\beta(1 \mathrm{ng} / \mathrm{mL})$, hTLR2 protein seemed to be down-regulated. However, hTLR4 protein appeared to be unchanged by exposure to IL- $1 \beta$. These observations suggest but do not actually determine that IL- $1 \beta$ stimulation may regulate hTLR2 protein expression inasmuch as measurements were qualitative.

Immunofluorescence analysis of hTLR2 and hTLR4 in H4 cells and fetal intestinal samples. To confirm that the expres- 
sion of hTLR2 and hTLR4 in H4 cells is recapitulated under in vivo conditions, 18- to 21-wk-old fetal ileal tissues were assessed by indirect immunofluorescence (Fig. 3). Indirect immunofluorescent staining with anti-hTLR2 and anti-hTLR4 antibodies showed immunoreactivity in both $\mathrm{H} 4$ cells and in the epithelium of the fetal small intestine (Fig. $3 A$ ). In addition, the expression of hTLR2 and hTLR4 were more pronounced in the basolateral aspect of the proliferative crypt epithelial compartment. However, weaker staining for hTLR2 and hTLR4 was also observed in the differentiated villus epithelium and on the microvillus surface. After $\mathrm{H} 4$ cells were exposed to IL-1 $\beta$ and LPS (Fig. $3 B$ ), a slight increase in hTLR2 immunofluorescence was noted with IL- $1 \beta$ and a slight decrease was apparent with LPS. No change in hTLR4 immunofluorescence was noted with IL- $1 \beta$, but it was slightly

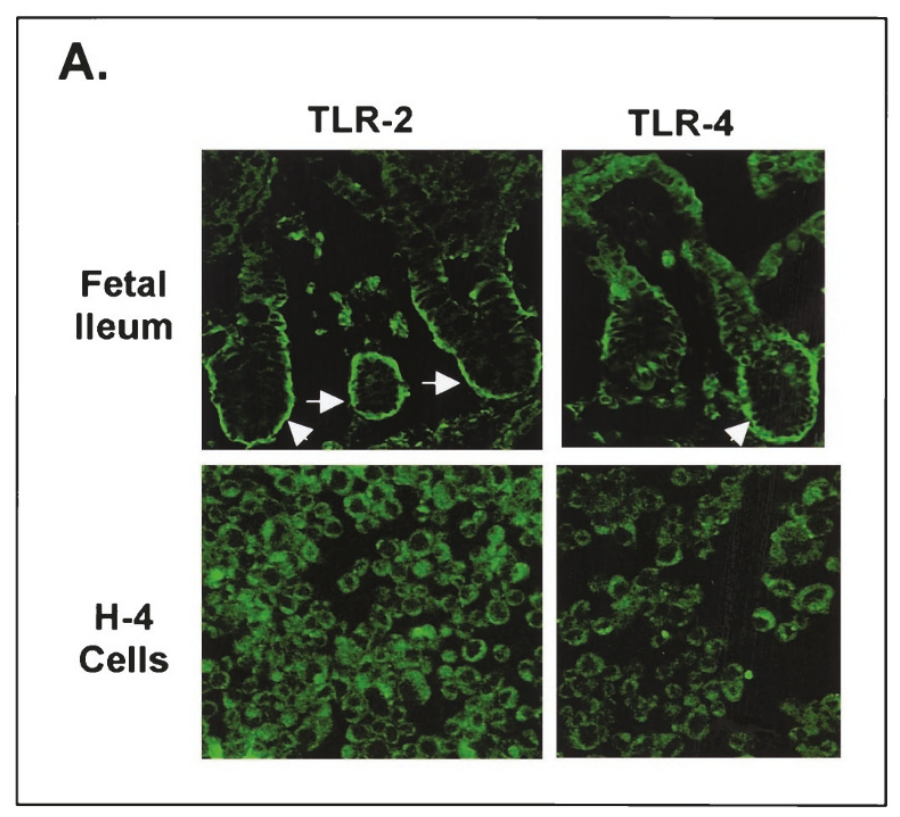

\section{B. TLR-2 expression in $\mathrm{H} 4$ cells}

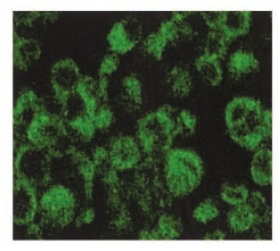

Basal

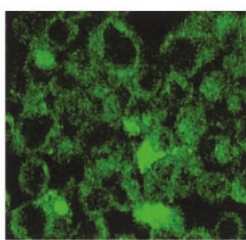

IL-1ß

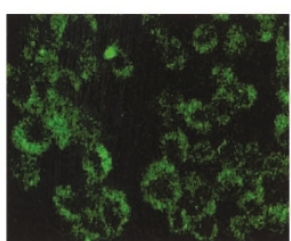

LPS
Figure 3. (A) Fetal ileal segments (20 wk old) exhibit strong hTLR2 and hTLR4 immunoreactivity on the basolateral surface of the proliferative crypt epithelium (arrow) and weaker staining on the villus epithelium. Lymphocytes in the mucosa also stained for hTLR2 and hTLR4 $(B)$. H4 cells exposed to IL-1 $\beta$ demonstrate a slight increase in hTLR2 immunostaining and a somewhat decreased staining after exposure to LPS. In contrast, H4 cells show a decrease in hTLR4 expression in response to LPS but show no change with IL-1 $\beta$ (data not shown). The degree of immunostaining is greater for hTLR2 when compared with hTLR4, whereas no immunoreactivity is observed with the control antibody (data not shown). H4 cells and fetal ileal sections are also counterstained for epithelial specific cytokeratin, CK5, which co-localized with hTLR2 and hTLR4 (data not shown). Results are representative of three indirect immunofluorescence studies. decreased with LPS (data not shown). These observations suggest but do not prove a possible specific regulation of the expression of hTLR2 and hTLR 4 protein by inflammatory stimuli. These results also differ from those seen for hTLR2 expression after IL-1 $\beta$ exposure in Western blot analysis.

\section{DISCUSSION}

In this study, we provide compelling evidence that hTLR2 and hTLR4 are expressed in the immature human intestine. This observation helps explain the capacity of immature human enterocytes to function as nonclassical immune cells in mounting an immediate, innate immune response to a PAMP, i.e. LPS, and underscores their importance as an active participant in mucosal barrier function to prevent invasion by luminal Gram-negative bacteria. This study was undertaken when tolllike receptors on lymphocytes were shown to activate cytokine transcription via a signal transduction pathway similar to that of the IL-1 receptor (10) leading to activation of the transcription factor NF $\kappa \mathrm{B}$ and up-regulation of IL-8, TNF- $\alpha$, and $\gamma$-interferon transcription (17).

Because endotoxin interaction with cultured intestinal epithelial cancer cell lines has been shown to up-regulate inflammatory cytokines such as IL-8 $(5,6)$, we assumed that the mechanism of activation may be similar to that reported for lymphocytes. If toll-like receptors existed on the surface of enterocytes to interact with the LPS-LBP-CD14 complex, this observation would explain the mechanism of action of LPS on enterocyte cytokine gene expression. Recently, Cario et al. (18) reported the presence of hTLR2 and hTLR4 mRNA and protein on several human colonic cancer cell lines, suggesting the possibility that hTLR on enterocytes may mediate the endotoxin response, as a PRR, in a manner similar to lymphoid cells.

In this study, we have shown by RT-PCR the presence of hTLR2 and hTLR4 mRNA in a fetal human primary intestinal epithelial cell line (H4 cells) and in fetal small intestinal ileal samples.

hTLR2 and hTLR4 protein were noted to be present principally on the basolateral surface of human fetal small crypt enterocytes (Fig. 3), although receptors were also noted on the microvillus surface and villous epithelium. As a partial explanation for this observation, several microbiologists have shown that Gram-negative organisms such as Salmonella typhi, Shigella flexneri, and Bacteroides fragilis secrete factors that may open tight junctions between enterocytes to allow for paracellular penetration of the organism or its LPS $(2,3,22)$. It is, therefore, entirely plausible that the breaching of the epithelial barrier after initial interaction with luminal pathogens and subsequent migration of these Gram-negative organisms between cells may allow LPS to interact with hTLR on the basolateral surface, which will not occur in an intact mucosal barrier. However, if the hTLR are predominantly located on the apical pole, they will constantly be exposed to luminal microbes and their PAMP will, thus, be in a state of perpetual activation. It is tempting to speculate that in mature enterocytes, especially from patients with inflammatory bowel disease, this may be so and perhaps provide a partial explanation 
for why, in these patients, the intestinal inflammation is persistent and unmitigated. However, additional studies are needed to support this hypothesis.

In previous studies, we have reported that inflammatory stimulants, e.g. LPS and IL- $1 \beta$, interacting with H4 cells or with fetal intestinal organ cultures resulted in a greater IL-8 enterocyte response than the same dose of these mediators interacting with either confluent Caco-2 cells (mature enterocytes) or biopsies of small intestine from older children (19). With the observation of this study that hTLR exist on human fetal enterocytes, we can now study whether receptor or postreceptor steps in the complete pathway of LPS stimulation of IL-8 accounts for the accentuated inflammatory response to microbial stimulation leading to necrotizing enterocolitis.

In these studies, hTLR expression on enterocytes as measured by RT-PCR (Fig. 1), Western blot analysis (Fig. 2), and immunohistochemistry (Fig. 3) seems to be regulated by exogenous and endogenous inflammatory stimuli (LPS and IL$1 \beta)$. RT-PCR measurements expressed per $\beta$-actin mRNA by densitometry (Fig. $1 B$ ) suggests that IL- $1 \beta$ may up-regulate hTLR2 and hTLR4 transcription and LPS may down-regulate hTLR4 transcription. However, the observations made by Western blot and immunofluorescent analysis are qualitative and contradictory and can only be interpreted as showing a change from constitutive expression. These observations are preliminary and will require additional studies to quantitate hTLR expression before a definitive conclusion can be reached. However, it is tempting to suggest that the downregulation of hTLR in response to LPS may be a means by which the host tolerates the presence of bacterial products in its intestinal lumen, thereby avoiding a sustained immunologic response to proinflammatory mediators that could cause deleterious effects to the gut. On the other hand, the up-regulation of the hTLR in response to activation by an endogenous proinflammatory stimulant, IL- $1 \beta$, may be necessary to cope with imminent invasion by pathogens.

In summary, this study demonstrates for the first time that hTLR2 and hTLR4 exist on human fetal enterocytes. It also suggests that the immature epithelium has the capacity to mount an acute, innate immune response to luminal pathogens and that intestinal epithelial cells play an important immunoregulatory role in protecting the host from noxious luminal stimuli.
Acknowledgment. The authors wish to thank Dr. Lei Lu for her technical assistance with the immunofluorescence studies.

\section{REFERENCES}

1. Sanderson IR, Walker WA 1999 Mucosal barrier: an overview. In: Ogra PL, Mestecky J, Lamm ME, Strober W, Bienenstock J, McGhee JR (eds) Handbook of Mucosal Immunology. Academic Press, New York, pp 5-17

2. Bloom PD, Boedecker EC 1996 Mucosal immune responses to intestinal bacterial pathogens. Semin Gastrointest Dis 7:151-166

3. Hromockyj AE, Falkow S 1999 Interactions of bacteria with the gut epithelium. In: Blaser MJ, Smith PD, Ravdin JI, Greenberg HB, Guerrant RL (eds) Infections of the Gastrointestinal Tract. Raven Press, New York, pp 603-615

4. Aderen A, Ulevitch RJ 2000 Toll-like receptors in the induction of the innate immune response. Nature 406:782-787

5. Stadnyk AW 1994 Cytokine production by epithelial cells. FASEB J 8:1041-1047

6. Eckkmann L, Jung HC, Schurer-Maly C, Panja A, Morzycka-Wrobleska E, Kagnoff MF 1993 Differential cytokine expression by human intestinal epithelial cell lines: regulated expression of interleukin-8. Gastroenterology 105:1689-1697

7. Kopp EB, Medzhitov R 1999 The toll-receptor family and control of innate immunity. Curr Opin Immunol 11:13-19

8. Medzhitov R, Janeway C 2000 Innate immunity. N Engl J Med 343:338-344

9. Anderson KV 2000 Toll signaling pathways in the innate immune response. Curr Opin Immunol 12:13-19

10. Medzhitov R, Preston-Hurlburt P, Janeway Jr CA 1997 A human homologue of the Drosophila Toll protein signals activation of adaptive immunity. Nature 388:394-397

11. Rock FL, Hardman G, Timans JC, Kastelein RA, Bazan JF 1998 A family of human receptors structurally related to Drosophila Toll. Proc Natl Acad Sci U S A 95:588593

12. Lien E, Sellati TJ, Yoshimura A, Flo TH, Rawadi G, Finberg RW, Ca JD, Espevik T, Ingalls RR, Radolf JD, Golenbock DT 1999 Toll-like receptor 2 functions as a pattern recognition receptor for diverse bacterial products. J Biol Chem 274:33419-33425

13. Chuang TH, Ulevitch RJ 2000 Cloning and characterization of a sub-family of human toll-like receptors: hTRL7, hTLR8 and hTLR9. Eur Cytokine Netw 11:372-378

14. Takeuchi O, Kawai T, Sanjo H, Copeland NG, Gilbert DJ, Jenkins NA, Takeda K, Akira S 1999 TLR6: a novel member of an expanding Toll-like receptor family. Gene 23:59-65

15. Yoshimura A, Lien E, Ingalls RR, Tuomanen E, Dziarski R, Golenbock DT 1999 Cutting edge recognition of Gram positive bacterial cell wall components by the innate immune system occurs via Toll-like receptors 2. J Immunol 163:1-5

16. Chow JC, Young DW, Golenbock DT, Christ WJ, Gusovsky SA 1999 Toll-like receptor-4 mediates lipopolysaccharide-induced signal transduction. J Biol Chem 274:10689-10692

17. Jobin C, Sartor BR 2000 The IkB/NF-kB system: a key determinant of mucosal inflammation and protection. Am J Physiol 278:C451-C462

18. Cario E, Rosenberg IM, Brandwein SL, Beck PL, Reinecker HC, Podolsky DK 2000 Lipopolysaccharide activates distinct signaling pathways in intestinal epithelial cell lines expressing Toll-like receptors. J Immunol 164:966-972

19. Nanthakumar N, Fusunyan RD, Sanderson IR, Walker WA 2000 Inflammation in the developing human intestine: a possible pathophysiologic basis for necrotizing enterocolitis. Proc Natl Acad Sci U S A 97:6043-6048

20. Sanderson IR, Ezzell RM, Kedinger M, Erlanger M, Xu Z, Pringault E, Leon-Robine S, Louvard D, Walker WA 1996 Human fetal enterocytes in vitro: modulation of the phenotype extracellular matrix. Proc Natl Acad Sci U S A 93:7717-7722

21. Zhang FX, Kirschning CJ, Mancinelli R, Xu XP, Jin YP, Faure E, Mantovani A, Rothe M, Muzio M, Arditi M 1999 Bacterial lipopolysaccharide activates nuclear factor- $\kappa \mathrm{B}$ signaling mediators in cultured human dermal endothelial cells and mononuclear phagocytes. J Biol Chem 274:7611-7614

22. McCormick BA, Gerwirtz AT, Madara JL 1998 Epithelial cross-talk with bacteria and immune cells. Curr Opin Gastroenterol 14:492-497 